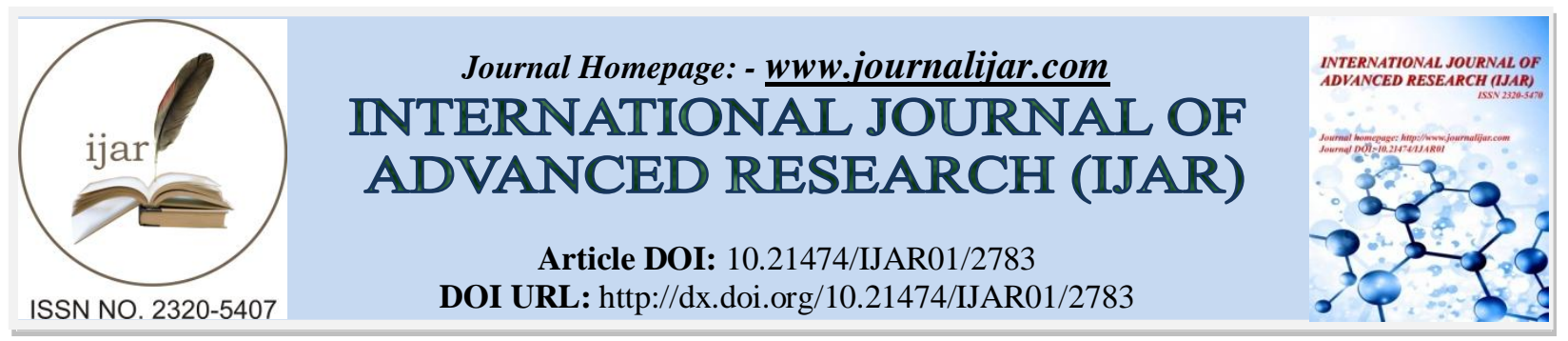

RESEARCH ARTICLE

\title{
ASPARTATE AMINOTRANSFERASE TO PLATELET RATIO INDEX VERSUS NEUTROPHIL TO LYMPHOCYTE RATIO FOR PREDICTION OF POST RADIOFREQUENCY ABLATION RECURRENCE OF HEPATOCELLULAR CARCINOMA.
}

Mohammad M. Sallam, Emad Hamed Fawzy and Hany Mohamed Elsadek . Internal Medicine Department, Faculty of Medicine, Zagazig University, Egypt.

\section{Manuscript Info}

.

\section{Manuscript History}

Received: 15 November 2016

Final Accepted: 17 December 2016

Published: January 2017

Key words:-

Hepatocellular carcinoma,

Radiofrequency ablation

\section{Abstract}

Background \& Aims: Tumor recurrence after curative radiofrequency ablation (RFA) of hepatocellular carcinoma (HCC) is common. The burden of frequent post- ablation investigations especially triphasic computerized tomography (TCT) is high. We investigated whether post ablation measurement of aspartate aminotransferase to platelet ratio index (APRI) and neutrophil to lymphocyte ratio (NLR) as simple and cheap biomarkers can precisely predict HCC recurrence and which of them is more valuable.

Methods: In this retrospective study, the demographic, clinical, laboratory and imaging data of 42 HCC patients treated with RFA were statistically analyzed. Patients were classified into two groups; those with HCC recurrence (group I) and those without (group II). In order to test the value of baseline NLR versus that of APRI in predicting tumor recurrence and compare both to alfa fetoprotein (AFP), we used receiver operating curve (ROC) statistics.

Results: Mean values of AST, Platelets count, APRI, NLR and AFP showed significant correlation with HCC recurrence. Using logistic regression analysis, NLR was the only independent risk factor predicting HCC recurrence. NLR had the highest sensitivity and specificity for prediction of HCC recurrence (90.9\% and 85\%) followed by that of AFP $(86.4 \%$ and $75 \%)$, and lastly by that of APRI $(72.7 \%$ and $70 \%)$.

Conclusion: NLR is a promising, simple and cheap surrogate marker for prediction of $\mathrm{HCC}$ recurrence after radiofrequency ablation, and is far more significant than APRI.

Copy Right, IJAR, 2016,. All rights reserved.

\section{Introduction:-}

Hepatocellular carcinoma (HCC) is the fifth most common cancer worldwide (1).HCC is a primary malignancy of the liver and occurs predominantly in patients with underlying chronic liver disease and cirrhosis (2). The incidence of HCC is highest in Asia and Africa, where the endemic high prevalence of hepatitis B (HBV) and hepatitis C (HCV) strongly predisposes to the development of chronic liver disease and subsequent development of HCC (3). Radiofrequency ablation (RFA) became a main modality of loco-regional therapy for HCC, because of its 
effectiveness and safety for small $\mathrm{HCC}(<5.0 \mathrm{~cm})$, with a 3 -year survival rate of $62-77 \%$, a low treatment complication rate of $8-9 \%$, and a low treatment mortality rate of $0-0.5 \%$ (4-6).

A number of factors, such as number and size of tumor nodules, increased levels of serum tumor markers, hepatitis $\mathrm{C}$ virus (HCV), HBV infections, diabetes mellitus and hyperglycemia, were reported to be related to $\mathrm{HCC}$ recurrence after RFA (7,8).

Despite the established role of alfa fetoprotein (AFP) in HCC diagnosis, it is of low significant value in prediction of $\mathrm{HCC}$ recurrence $(\mathbf{9 , 1 0})$.

Aspartate aminotransferase(AST)-to-platelet ratio index (APRI) is a simple and feasible test validated in assessing the stage of fibrosis and in predicting prognosis for patients with chronic hepatitis (11,12).APRI shows reliable discriminative ability for predicting not only overall survival of HCC patients, but also tumor recurrence (13).APRI was also found to be a prognostic biomarker in small HCC patients after RFA therapy and surgical resection $(\mathbf{1 4 , 1 5 )}$.

Some studies demonstrated that background liver inflammation and fibrosis play important roles both in the process of hepatocarcinogenesis and in recurrence after resection surgery (16,17). Moreover, there are increasing evidences that the presence of systemic inflammation correlates with poorer cancer-specific survival in certain cancers (1820).Various markers of systemic inflammatory response, including cytokines, C-reactive protein (CRP), and absolute blood neutrophil or lymphocyte count as well as their ratio such as neutrophil-to-lymphocyte ratio (NLR) have been investigated for their prognostic roles in certain cancer populations $(\mathbf{2 1 , 2 2})$.Patients with elevated NLR have a relative lymphocytopenia and neutrophilic leucocytosis which denote that the balance is tipped in favor of protumor inflammatory response and is associated with poor oncologic outcome (23).Significant elevation of NLR increases the risk of $\mathrm{HCC}$ recurrence and recipient death in patients undergoing transplantation for HCC (24).

Several studies indicate that serum AFP level, AST level, AST-to-alanine aminotransferase (ALT) ratio, APRI and NLR have been associated with HCC recurrence and poor survival (11,12,25-27).

We conducted this study to compare the value of NLR and APRI for prediction of post RFA recurrence of HCC.

\section{Patients and Methods:-}

This retrospective study had been carried out in Internal Medicine Department, Gastroenterology Unit, Faculty of Medicine, Zagazig University, from Aug. 2014 to Aug. 2016. All procedures performed were in accordance with the ethical standards of the institutional research committee and with the Helsinki Declaration and its later amendments.

Inclusion criteria : Patients with single focal lesion $\leq 5 \mathrm{~cm}$ or up to three lesions each $\leq 3 \mathrm{~cm}$ of HCC who received curative RFA.

Exclusion criteria included portal vein thrombosis, extra hepatic metastasis, class C liver cirrhosis (according to Child-Turcotte-Pugh score) $\mathbf{( 2 8 , 2 9 )}$. Also, we excluded patients with hematological disorders, active infection, heart failure, renal impairment (serum creatinine $>1.5 \mathrm{mg} / \mathrm{dl}$ ), pregnancy or history of drug abuse or ongoing chemotherapy intake and those who lost follow up.

Out of 50 HCC patients who underwent curative RFA, 42 patients who met our inclusion and exclusion criteria were selected and enrolled in the study. The studied 42 patients were divided into two groups; group I (recurrence group) including 22 patients with post-ablation recurrence of $\mathrm{HCC}$, and group II (recurrence free group) including 20 patients without recurrence. Diagnosis of recurrence was made by radiologic evidence using triphasic CT (TCT).

All studied patients underwent baseline (one month after curative RFA) clinical examination, imaging studies including chest $\mathrm{x}$ ray, pelviabdominal ultrasonography and TCT, as well as laboratory investigations including complete blood count (CBC), liver function tests, kidney function tests, coagulation profile and serum AFP level, in addition to calculation of APRI and NLR. All participants were subjected to follow up reevaluation of AFP, APRI and NLR every 3 months for 2 years. And TCT every 6-12 months.

\section{Statistical Analysis:-}

The quantitative variables were expressed as means \pm standard deviation (SD) and the categorical variables as count numbers and proportions. Statistical analysis was performed with SPSS package version 19 (SPSS Inc., Chicago, IL) using the suitable test e.g. ANOVA, qui square, Pearson's correlation and logistic regression analysis. The result was 
considered significant if the $P \leq 0.05$. In order to test the predictive accuracy (sensitivity and specificity) of various markers in predicting tumor recurrence, we used receiver operating curve (ROC) statistics.

\section{Results:-}

Table (1) showed comparison between group I and group II regarding demographic, clinical and laboratory parameters. There were significant differences between the two groups regarding the investigated tumor markers; APRI, NLR and AFP. HCC recurrence showed a significant positive correlation with each of AST, APRI, NLR and AFP, and a significant negative correlation with platelets count (table 2).

Using logistic regression analysis model, high NLR was the independent risk factor $(p=0.002)$ for prediction of HCC recurrence(table 3). In order to test the predicting accuracy (sensitivity and specificity) of NLR in predicting tumor recurrence as compared to APRI and AFP, we used ROC curve statistics (table 4). NLR had the highest sensitivity and specificity (90.9 \% and $85 \%)$ followed by AFP $(86.4 \%$ and $75 \%)$, and lastly by APRI (72.7\% and $70 \%$ ) (table 4, fig.1, fig. 2, fig. 3).

Table 1:- Demographic, clinical and laboratory parameters of all participants.

\begin{tabular}{|c|c|c|c|c|}
\hline Parameters & $\begin{array}{l}\begin{array}{l}\text { All patients } \\
(\mathrm{n}=42)\end{array} \\
\end{array}$ & $\begin{array}{l}\text { Group I } \\
(\mathrm{n}=22)\end{array}$ & \begin{tabular}{|l} 
Group II \\
$(\mathrm{n}=20)$
\end{tabular} & $\mathbf{P}$ \\
\hline Age (mean $\pm S D$, years) & $60.24 \pm 10.30$ & $58.14 \pm 9.61$ & $62.55 \pm 10.78$ & NS \\
\hline Male Gender(n,\%) & $26, \quad 61.90$ & $15, \quad 68.20$ & $11 \pm 55.00$ & NS \\
\hline Lesion(s) number (mean \pm SD) & $1.29 \pm 0.64$ & $1.32 \pm 0.72$ & $1.25 \pm 0.55$ & NS \\
\hline Lesion(s) size $($ mean $\pm \mathrm{SD}, \mathrm{cm})$ & $3.25 \pm 1.19$ & $3.38 \pm 1.19$ & $3.12 \pm 1.05$ & NS \\
\hline \multicolumn{5}{|l|}{ Possible HCC etiology } \\
\hline HCV infection $(\mathrm{n}, \%)$ & $30, \quad 71.40$ & $17, \quad 77.30$ & $13 \pm 65.00$ & NS \\
\hline HBV infection (n, \%) & $10, \quad 23.80$ & 22.70 & $5 \pm 25.00$ & NS \\
\hline Non-viral (n, \%) & 9.50 & 9.10 & $2 \pm 10.00$ & NS \\
\hline Cirrhosis (n, \%) & $39, \quad 92.90$ & $20 . \quad 90.90$ & $19 \pm 95.00$ & NS \\
\hline \multicolumn{5}{|l|}{ Child's Class } \\
\hline $\mathrm{A}(\mathrm{n}, \%)$ & $35, \quad 83,30$ & $19, \quad 86.40$ & $16 \pm 80.00$ & NS \\
\hline $\mathrm{B}(\mathrm{n}, \%)$ & 16.70 & $3, \quad 13.40$ & $4 \pm 20.00$ & NS \\
\hline Antiviral therapy (n, \%) & $15, \quad 35.70$ & $7, \quad 31.80$ & $8 \pm 20.00$ & NS \\
\hline $\mathbf{A S T}(\operatorname{mean} \pm \mathrm{SD}, \mathrm{IU})$ & $1.66 \pm 0.72$ & $1.86 \pm 0.80$ & $1.43 \pm 0.56$ & NS \\
\hline Platelet count $\left(\right.$ mean $\left.\pm \mathrm{SD}, \times 10^{9} / \mathrm{L}\right)$ & $112.79 \pm 64.59$ & $95.27 \pm 45.34$ & $132.05 \pm 77.36$ & NS \\
\hline APRI $($ mean \pm SD $)$ & $1.82 \pm 0.98$ & $2.12 \pm 0.74$ & $1.49 \pm 1.12$ & 0.036 \\
\hline NLR $($ mean \pm SD) & $1.78 \pm 0.97$ & $2.37 \pm 0.82$ & $1.13 \pm 0.67$ & $<0.001$ \\
\hline AFP $($ mean \pm SD, $\mathrm{ng} / \mathrm{ml})$ & $3818 \pm 6053$ & $6107 \pm 6655$ & $1300 \pm 4168$ & 0.008 \\
\hline
\end{tabular}

NS: non significant.

Table 2:- Correlation between different clinicopathologic parameters and tumor recurrence.

\begin{tabular}{|l|l|l|}
\hline Parameter & r & P \\
\hline Age & -0.217 & NS \\
\hline Sex & 0.136 & NS \\
\hline Lesion(s) number & 0.054 & NS \\
\hline Lesion(s) size & 0.118 & NS \\
\hline HCC etiology & -0.031 & NS \\
\hline Cirrhosis & -0.079 & NS \\
\hline Child's Class & -0.085 & NS \\
\hline Antiviral therapy & -0.085 & NS \\
\hline AST & 0.299 & 0.027 \\
\hline Platelet count & -0.288 & 0.032 \\
\hline APRI & 0.325 & 0.018 \\
\hline NLR & 0.648 & $<0.001$ \\
\hline AFP & 0.401 & 0.004 \\
\hline
\end{tabular}

NS: non significant. 
Table 3:- Independent risk factor(s) predicting tumor recurrence using logistic regression analysis.

\begin{tabular}{|l|l|l|l|}
\hline Predicting factor & \multirow{2}{*}{} & \multicolumn{2}{|l|}{$\mathbf{9 5 \%}$ Confidence Interval for B } \\
\cline { 3 - 4 } & & Lower bound & Upper bound \\
\hline AST & NS & -0.125 & 0.524 \\
\hline Platelet count & NS & -0.007 & 0.002 \\
\hline APRI & NS & -0.499 & 0.060 \\
\hline NLR & 0.002 & 0.121 & 0.519 \\
\hline AFP & NS & 0.000 & 0.000 \\
\hline
\end{tabular}

NS: non significant.

Table 4:- Comparison between sensitivity and specificity of APRI, NLR and AFP in predicting tumor recurrence using ROC curve statistics

\begin{tabular}{|c|c|c|c|c|c|c|c|}
\hline \multirow[t]{2}{*}{ Predictor } & \multirow[t]{2}{*}{ Cut-off value } & \multirow[t]{2}{*}{$\begin{array}{ll}\text { Area } & \text { under } \\
\text { the } & \text { curve } \\
(\mathrm{AUC}) & \end{array}$} & \multicolumn{2}{|c|}{$\begin{array}{l}95.0 \% \\
\text { Confidence } \\
\text { Interval (CI) }\end{array}$} & \multirow[t]{2}{*}{$\mathbf{P}$} & \multirow[t]{2}{*}{ Sensitivity } & \multirow[t]{2}{*}{ Specificity } \\
\hline & & & $\begin{array}{l}\text { Lower } \\
\text { bound }\end{array}$ & $\begin{array}{l}\text { Upper } \\
\text { bound }\end{array}$ & & & \\
\hline APRI & 1.66 & 0.744 & 0.581 & 0.907 & 0.007 & $72.7 \%$ & $70.0 \%$ \\
\hline NLR & 1.55 & 0.903 & 0.801 & 1.000 & $<0.001$ & $90.9 \%$ & $85.0 \%$ \\
\hline $\mathbf{A F P}(\mathrm{ng} / \mathrm{ml})$ & 210 & 0.864 & 0.745 & 0.982 & $<0.001$ & $86.4 \%$ & $75.0 \%$ \\
\hline
\end{tabular}

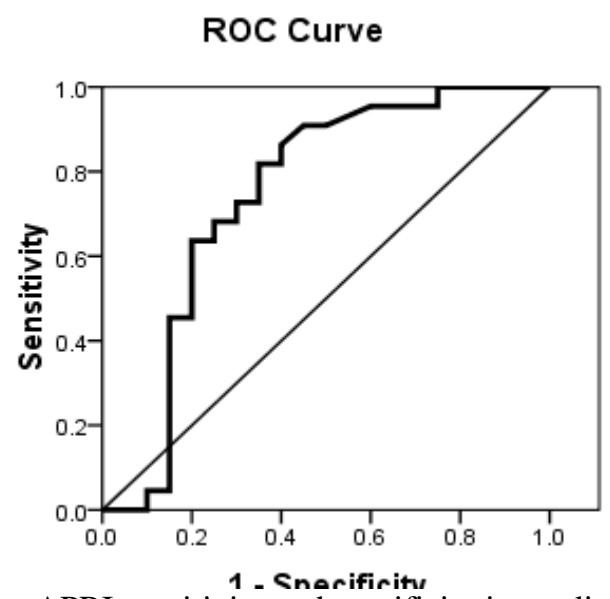

Figure 1:- ROC Curve for APRI sensitivity and specificity in predicting tumor recurrence

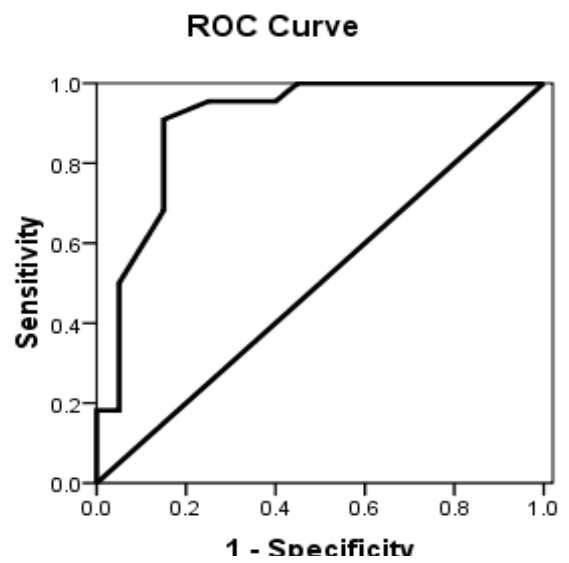

Figure 2:- ROC Curve for NLR sensitivity and specificity in predicting tumor recurrence 


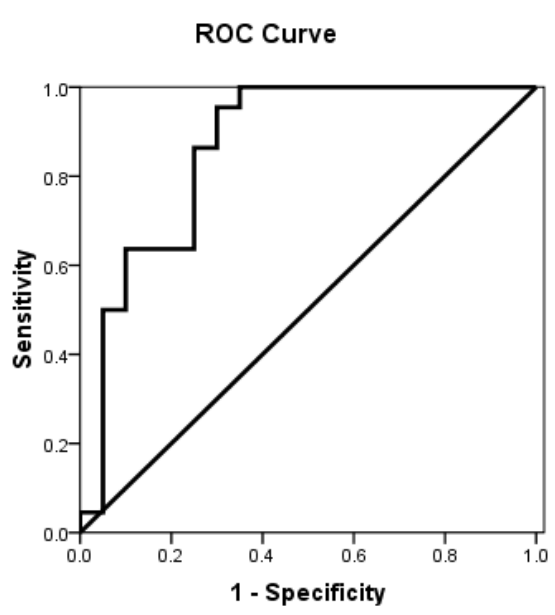

Figure 3:- ROC Curve for AFP sensitivity and specificity in predicting tumor recurrence

\section{Discussion:-}

The burden of HCC has been increasing in Egypt with a doubling in the incidence rate in the past 10 years (30).Several studies investigated predictors for prognosis and survival for patients of HCC after RFA but only few studies investigated the predictors of tumor recurrence after radiofrequency $(\mathbf{3 1}, \mathbf{3 2})$.

This study was conducted for evaluation of the postoperative NLR and APRI as predictors of post RFA recurrence of HCC. Both markers were compared together and to AFP. The mean values of the investigated markers (APRI, NLR and AFP) were significantly higher in the recurrence group than in the recurrence free group $(2.12 \pm 0.74$ versus $1.49 \pm 1.12, P=0.036 ; 2.37 \pm 0.82$ versus $1.13 \pm 0.67, P<0.001$ and $6107 \pm 6655$ versus $1300 \pm 4168, P=0.008$, respectively).

In the current study, five parameters (AST, PLT, APRI, NLR and AFP) showed significant correlation with HCC recurrence ( $P$ values were: $0.027,0.032,0.018,<0.001$ and 0.004 , respectively).

In this study, APRI showed a significant positive correlation to HCC recurrence ( $\mathrm{r}=0.325, P=0.018)$. Similarly, APRI was validated in several previous studies as a simple, noninvasive way to assess the degree of liver fibrosis in patients with chronic hepatitis $\mathrm{B}$ or $\mathrm{C}$ and also had a reliable discriminative ability for predicting overall survival and $\mathrm{HCC}$ recurrence $(\mathbf{1 4 , 3 3 , 3 4 )}$.

In our study, NLR showed a highly significant positive correlation to HCC recurrence $(\mathrm{r}=0.648, P<0.001)$. By using logistic regression analysis model, the only independent risk factor for prediction of tumor recurrence among the above five parameters was NLR $(P=0.002)$.

It is widely accepted that inflammatory process plays a significant role in several stages of tumor development and progression.The tumor increases the inflammatory process, which in turn predisposes to tumor progression, via inhibition of apoptosis and promotion of angiogenesis $(\mathbf{2 1 , 2 2})$. Wu and colleagues reported that hepatic inflammatory activity was associated with early HCC recurrence (16).

NLR, a biomarker of tumor inflammation and host immunity, was associated with increased mortality in cancer (35).In agreement with the results of our study Chen et al.(36), Dan et al.(37) and Tajiriet al.(38) reported that high postoperative NLR is associated with high recurrence rate of HCC after RFA.

The association between the high level of NLR and the increased numbers of HCC recurrence in our study and in previous studies may be attributed to the fact that host's immune response to tumors depends on lymphocytes, whereas patients with a large NLR have relative lymphocytopenia, which results in the attenuation of lymphocytemediated antitumor immune responses in these patients (36). Another explanation is that the patients with a large NLR usually have an enhanced neutrophil response, which could promote the production of pro-angiogenic factors, 
including vascular endothelial growth factor, interleukin-8, and matrix metalloproteinase. These pro-angiogenic factors may promote tumor growth and recurrence (39).

The comparison between sensitivity and specificity of APRI, NLR and AFP in predicting tumor recurrence, in our study, using ROC curve statistics revealed that NLR attained the highest level of sensitivity and specificity $(90.9 \%$ and $85 \%$ ) with Cut-off value (1.55) compared to AFP (86.4\% and 75\%) and APRI (72.7\% and 70\%).

In contrary to our results, in their study on 98 post RFA HCC patients, Chung et al.(40) reported that APRI was significantly higher in the recurrence group than in the recurrence free group $(2.3 \pm 1.8$ vs. $1.3 \pm 1.4, P=0.018)$, while there was no significant difference between the two studied groups regarding NLR. The difference between our results and that of Chung et al. may be attributed to the different etiologic background of HCC as in our current study the main etiology of HCC was chronic HCV infection (77.3\%) while in study of Chung et al., the main etiology was chronic HBV infection (55.6\%).

\section{Conclusion:-}

From the aforementioned findings, we can speculate that NLR is more valuable than APRI in predicting HCC recurrence after RFA .NLR is a promising surrogate marker in predicting post RFA HCC recurrence .Further studies are needed to validate the clinical relevance of NLR in follow up of HCC patients.

\section{References:-}

1. Llovet JM, Burroughs A, Bruix J. Hepatocellular carcinoma. Lancet 2003; 362(9399): 1907-1917.

2. Alison MR. Liver stem cells: Implications for hepatocarcinogenesis. Stem Cell Rev. 2005; 1(3):253-60.

3. Llovet JM, Fuster J, Bruix J. The Barcelona approach: diagnosis, staging, and treatment of hepatocellular carcinoma. Liver Transpl. 2004; 10(Suppl 1):S115-20.

4. Mulier S, Mulier P, Ni Y, et al. Complications of radiofrequency coagulation of liver tumours. Br J Surg 2002; 89: 1206-1222.

5. Lau WY, Leung TW, Yu SC, Ho SK .Percutaneous local ablative therapy for hepatocellular carcinoma: a review and look into the future. Ann Surg 2003;237:171-179.

6. Chen MS, Li JQ, Zheng Y, et al. A prospective and omized trial comparing percutaneous local ablative therapy and partial hepatectomy for small hepatocellular carcinoma. Ann Surg 2006; 243: 321-328.

7. Shiina S, Tateishi R, Arano T, et al. Radiofrequency ablation for hepatocellular carcinoma: 10-year outcome and prognostic factors. Am.J. Gastroenterol. 2012; 107: 569-77; quiz 78.

8. Hosokawa T, Kurosaki M, Tsuchiya K, et al. Hyperglycemia is a significant prognostic factor of hepatocellular carcinoma after curative therapy. World J. Gastroenterol.2013; 19: 249-57.

9. Yang X, Zhang Y, Zhang L, et al. Silencing alpha fetoprotein expression induces growth arrest and apoptosis in human hepatocellular cancer cell. Cancer Lett 2008; 271:281-293.

10. Arrieta O, Cacho B, Morales-Espinosa D, et al. The progressive elevation of alpha fetoprotein for the diagnosis of hepatocellular carcinoma in patients with liver cirrhosis. BMC Cancer 2007; 8:28.

11. Nunes D, Fleming C, Offner G, et al. Non invasive markers of liver fibrosis are highly predictive of liverrelated death in a cohort of $\mathrm{HCV}$-infected individuals with and without HIV infection. Am J Gastroenterol. 2010; 105:1346-1353.

12. Su CW, Chan $\mathrm{CC}$, Hung $\mathrm{HH}$, et al. Predictive value of aspartate aminotransferase to alanine aminotransferase ratio for hepatic fibrosis and clinical adverse outcomes in patients with primary biliary cirrhosis. $\mathrm{J}$ Clin Gastroenterol 2009; 43:876-883.

13. Wei-Yu Kao, Yi-You Chiou, Hung-Hsu H, et al. Risk factors for long-term prognosis in hepatocellular carcinoma after radiofrequency ablation therapy: the clinical implication of aspartate aminotransferase-platelet ratio index. European Journal of Gastroenterology \&Hepatology 2011; 23:528-536.

14. Shen S-L, Fu S-J, Chen B, et al. Preoperative aspartate aminotransferase to platelet ratio is an independent prognostic factor for hepatitis B-induced hepatocellular carcinoma after hepatic resection. Ann Surg Oncol. 2014;21(12):3802-9.

15. Kao W-Y, Chiou Y-Y, Hung H-H, et al. Risk factors for long-term prognosis in hepatocellular carcinoma after radiofrequency ablation therapy: the clinical implication of aspartate aminotransferase- platelet ratio index. Eur J Gastroenterol Hepatol. 2011;23(6):528-36.

16. Wu JC, Huang YH, Chau GY, et al. Risk factors for early and late recurrence in hepatitis B-related hepatocellular carcinoma.J Hepatol 2009; 51:890-897. 
17. Hung HH, Su CW, Lai CR, et al. Fibrosis and AST to platelet ratio index predict post-operative prognosis for solitary small hepatitis B related hepatocellular carcinoma. Hepatol. Int. 2010;4:691-699.

18. Jung MR, Park YK, Jeong O, et al. Elevated preoperative neutrophil to lymphocyte ratio predicts poor survival following resection in late stage gastric cancer. J. Surg. Oncol. 2011;104: 504-510.

19. Zahorec R. Ratio of neutrophil to lymphocyte counts-rapid and simple parameter of systemic inflammation and stress in critically ill. Bratisl Lek Listy 2001;102:5-14.

20. Thavaramara T, Phaloprakarn C, Tangjitgamol S, Manusirivithaya S. Role of neutrophil to lymphocyte ratio as a prognostic indicator for epithelial ovarian cancer. J Med Assoc Thai 2011; 94: 871-877.

21. Oh BS, Jang JW, Kwon JH, et al. Prognostic value of C-reactive protein and neutrophil-to-lymphocyte ratio in patients with hepatocellular carcinoma. BMC Cancer 2013; 13: 78.

22. Morris-Stiff G, Gomez D, Prasad KR. C-reactive protein in liver cancer surgery. Eur J Surg Oncol 2008; 34 : 727-729.

23. Hashimoto K, Ikeda $\mathrm{Y}$, Korenaga D, et al. The impact of preoperative serum C-reactive protein on the prognosis of patients with hepatocellular carcinoma. Cancer 2005; 103: 1856-1864.

24. Halazun KJ, Hardy M, Rana A. Negative impact of neutrophil lymphocyte ratio on outcome after liver transplantation for hepato-cellular carcinoma.Ann Surg 2009; 250: 141- 51.

25. Ng KK, Poon RT, Lo CM, et al. Analysis of recurrence pattern and its influence on survival outcome after radiofrequency ablation of hepatocellular carcinoma. J Gastro intest Surg 2008; 12:183-191.

26. N'Kontchou G, Mahamoudi A, Aout M, et al. Radiofrequency ablation of hepatocellular carcinoma: long-term results and prognostic factors in 235 Western patients with cirrhosis.Hepatology 2009; 50:1475-1483.

27. Tsai MC, Wang JH, Hung CH, et al. Favorable alpha-fetoprotein decrease as a prognostic surrogate in patients with hepatocellular carcinoma after radiofrequency ablation. J Gastroenterol Hepatol 2010; 25:605-612.

28. Durand F, Valla D. Assessment of the prognosis of cirrhosis: Child-Pugh versus MELD. J Hepatol 2005;42(suppl):S100-S107.

29. Pugh RN, Murray-Lyon IM, Dawson JL, Pietroni MC. Transection of the oesophagus for bleeding oesophageal varices. Br J Surg 1973;60:646-649.

30. Gomaa AI, Khan SA, Toledano MB, et al. Hepatocellular carcinoma: Epidemiology, risk factors and pathogenesis. World J Gastroenterol 2008; 14(27): 4300-4308.

31. El-Serag HB, Rudolph KL. Hepatocellular carcinoma: epidemiology and molecular carcinogenesis. Gastroenterology. 2007;132(7):2557-76.

32. Ji F, Fu SJ, Shen SL, et al. The prognostic value of combined TGF- $\beta 1$ and ELF in hepatocellular carcinoma.BMC Cancer 2015;15(1):116.

33. Ma J, Jiang Y, Gong G. Evaluation of seven noninvasive models in staging liver fibrosis in patients with chronic hepatitis B virus infection. Eur J Gastroenterol Hepatol. 2013;25(4):428-34.

34. Boursier J, Brochard C, Bertrais S, et al. Combination of blood tests for significant fibrosis and cirrhosis improves the assessment of liver-prognosis in chronic hepatitis C. Aliment Pharmacol Ther. 2014;40(2):17888.

35. Lai Q, Castro Santa E, Rico Juri JM,et al. Neutrophil and platelet-to-lymphocyte ratio as new predictors of dropout and recurrence after liver transplantation for hepatocellular cancer. Transpl Int 2014; 27: 32-41.

36. Chen TM, Lin CC, Huang PT, Wen CF. Neutrophil-to-lymphocyte ratio associated with mortality in early hepatocellular carcinoma patients after radiofrequency ablation. J Gastroenterol Hepatol 2012; 27: 553-561.

37. Dan J, Zhang Y, Peng Z, et al. Postoperative Neutrophil-to-Lymphocyte Ratio Change Predicts Survival of Patients with Small Hepatocellular Carcinoma Undergoing Radiofrequency Ablation. PloS One 2013;8(3):e58184.

38. Tajiri K, Baba H, Kawai K, et al. Neutrophil-to-lymphocyte ratio predicts recurrence after radiofrequency ablation in hepatitis B virus infection. J Gastroenterol Hepatol. $2016 \mathrm{Jul} ; 31(7): 1291-9$

39. Fondevila C, Metges JP, Fuster J, et al.p53 and VEGF expression are independent predictors of tumour recurrence and survival following curative resection of gastric cancer. Br J Cancer 2004; 90: 206-215.

40. Chung HA, Kim JH, Hwang Y, et al. Non invasive fibrosis marker can predict recurrence of hepatocellular carcinoma after radiofrequency ablation. Saudi J Gastroenterol 2016;22:57-63. 